\title{
Expression and clinical significance of glucose transporter-1 in pancreatic cancer
}

\author{
KAI LU $^{1 *}$, JIAN YANG $^{1 *}$, DE-CHUN LI ${ }^{1 *}$, SONG-BING HE ${ }^{1 *}$, DONG-MING ZHU ${ }^{1}$, \\ LI-FENG ZHANG ${ }^{1}$, XU ZHANG ${ }^{1}$, XIAO-CHEN CHEN ${ }^{2}$, BING ZHANG ${ }^{3}$ and JIAN ZHOU ${ }^{1}$ \\ ${ }^{1}$ Department of General Surgery, The First Affiliated Hospital of Soochow University, Suzhou, Jiangsu 215006; \\ ${ }^{2}$ Department of Pathology, Obstetrics and Gynecology Hospital of Fudan University, Shanghai 200090; \\ ${ }^{3}$ Department of Nuclear Medicine, The First Affiliated Hospital of Soochow University, Suzhou, Jiangsu 215006, P.R. China
}

Received March 28, 2015; Accepted April 22, 2016

DOI: $10.3892 / 01.2016 .4586$

\begin{abstract}
Increasing evidence has demonstrated that malignant cells exhibit increased glucose uptake, which facilitates survival and growth in a hypoxic environment. The glucose transporter-1 (GLUT-1) is overexpressed in a variety of malignant tumors. However, the association between GLUT-1 expression and clinicopathological factors, ${ }^{18} \mathrm{~F}$-fluorodeoxyglucose uptake and tumor proliferation in pancreatic cancer has not been investigated to date. In the present study, the expression of GLUT-1 in 53 pancreatic cancer tissues was analyzed, which revealed that GLUT-1 was overexpressed in pancreatic tissue and correlated with poor prognosis and clinicopathological characteristics, including increased tumor size, clinical stage and lymph node metastasis, maximum standardized uptake value ( $\mathrm{SUV}_{\max }$ ) and $\mathrm{Ki}-67$ expression. The receiver operating characteristic curve analysis indicated that a cut-off $\mathrm{SUV}_{\max }$ value of 4.830 was associated with optimal sensitivity $(88 \%)$ and specificity $(71.4 \%)$ for the detection of strong positive GLUT-1 expression. In addition, as the expression of GLUT-1 was found to correlate with Ki-67 expression, GLUT-1 may exhibit a significant effect on cell proliferation in pancreatic cancer. Overall, these findings indicate that GLUT-1 may represent a prognostic indicator, and a potential therapeutic target for pancreatic cancer.
\end{abstract}

\section{Introduction}

Pancreatic cancer remains one of the most lethal malignancies worldwide, with a high malignant potential and a poor

Correspondence to: Dr Jian Zhou, Department of General Surgery, The First Affiliated Hospital of Soochow University, 188 Shizi Street, Suzhou, Jiangsu 215006, P.R. China

E-mail: zhoujian06@suda.edu.cn

${ }^{*}$ Contributed equally

Key words: glucose transporter-1, ${ }^{18} \mathrm{~F}$-fluorodeoxyglucose, prognosis, Ki-67, pancreatic cancer prognosis. The number of new cases of pancreatic cancer was almost 48,960 in 2015. It is the fourth most common cause of cancer-associated mortality in Western society, with a median survival of $<6$ months and a 5-year survival rate of $5 \%(1,2)$. Despite advances in cancer therapy, pancreatic cancer is unresponsive to the majority of treatments $(3,4)$. To date, no targeted therapy to improve the clinical outcome has been identified. Consequently, development of molecular prognostic factors to improve patient selection for novel therapeutic approaches is urgently required.

Tumor hypoxia is a common phenomenon in solid tumors, and is associated with poor prognosis in several types of cancer, including laryngeal squamous cell carcinoma, ovarian cancer, breast cancer, gallbladder cancer and pancreatic cancer (5). Hypoxia leads to genetic instability and failure of DNA repair, which results in the selection of tumor cells toward a more aggressive phenotype. Under hypoxic conditions, tumor cells switch from oxygen-dependent glucose metabolism to anaerobic glycolysis (6). This cellular adaptation to hypoxia, known as the Warburg effect, is supported by an observed increase in glucose transport and consumption (7). High rates of glucose uptake and glycolysis supply the energy required for proliferation of malignant cells and tumor growth.

The glucose transporter (GLUT) family has been identified as belonging to the solute carrier 2A family (SLCZA). The members of this family differ in their affinity for glucose and their effects on physiological regulation $(8,9)$. Glucose transporter-1 (GLUT-1) is a member of the GLUT family, which is expressed in erythrocytes, endothelial cells, placenta and blood-tissue barriers, including the blood-brain and blood-nerve barriers $(10,11)$. Recent studies have demonstrated that GLUT-1 is often upregulated in various malignant tumors, including colorectal cancer (12), esophageal cancer (13), oral squamous cell carcinoma (14), renal cell carcinoma (15) breast cancer and lung cancer (16). It is also considered to be the predominantly elevated glucose transporter under ischemic and hypoxic conditions, whereby cells require glycolysis as an energy source. Positron emission tomography (PET) with ${ }^{18} \mathrm{~F}$-fluorodeoxyglucose $\left({ }^{18} \mathrm{~F}\right.$-FDG $)$ is a non-invasive diagnostic and prognostic tool used to evaluate the hypoxic status of tumors. The expression of glucose transporter proteins, in 
particular GLUT-1, is hypothesized to be associated with FDG uptake (17).

In the present study, immunohistochemical analysis was used to determine the level of GLUT-1 expression in human pancreatic cancer tissues and to evaluate the association between GLUT-1 expression and clinicopathological characteristics and prognosis. In addition, the association between GLUT-1 expression, ${ }^{18} \mathrm{~F}-\mathrm{FDG}$ accumulation and $\mathrm{Ki}-67$ expression was also investigated.

\section{Materials and methods}

Clinical data. The study sample was comprised of 53 formalin-fixed and paraffin-embedded pancreatic cancer tissue specimens and adjacent healthy tissues obtained from patients with pancreatic cancer. All patients underwent surgical resection at the First Affiliated Hospital of Soochow University (Suzhou, China) between January 2010 and December 2011. Patient characteristics and tumor status are summarized in Table I. The clinical stage was classified according to the seventh edition of the TNM classification of the American Joint Committee on Cancer (18). Patients that had received preoperative chemo-, radio-or immunotherapy were excluded. The study was conducted in accordance with the Declaration of Helsinki (19) and was approved by the Ethics Committee of Soochow University.

Immunohistochemistry (IHC). The samples were fixed with formalin (GE Healthcare Life Sciences, Logan, UT, USA) embedded in paraffin (GE Healthcare Life Sciences) and sectioned. Serial sections $(4-\mu \mathrm{m})$ subjected to immunohistological staining were fixed with freshly prepared $3 \% \mathrm{H}_{2} \mathrm{O}_{2}$ with $0.1 \%$ sodium azide to block endogenous peroxidase activity and treated with antigen retrieval solution (GE Healthcare Life Sciences) for $15 \mathrm{~min}$. After placing in blocking reagent (Roche Diagnostics, Basel, Switzerland) for $15 \mathrm{~min}$, the sections were incubated with primary rabbit monoclonal anti-GLUT-1 (dilution, 1:300; catalog no., ab115730; Abcam, Cambridge, MA, USA) or mouse monoclonal anti-Ki-67 (dilution, 1:500; catalog no., ab6526; Abcam) antibody overnight at $4^{\circ} \mathrm{C}$, followed by incubation with horseradish peroxidase-conjugated polyclonal goat anti-rabbit IgG secondary antibody (dilution, 1:500; catalog no., ab97200; Abcam) for $2 \mathrm{~h}$ at $4^{\circ} \mathrm{C}$. The signal was visualized by 3,3'-diaminobenzidene (Sangon Biotech Co., Ltd, Shanghai, China).

Evaluation of IHC. GLUT-1 expression was evaluated by light microscopy (Leica Microsystems, Mannheim, Germany) for immunostaining intensity and staining percentage. A total of 3 fields of view were examined at magnification, $x 200$. The staining intensity was classified as follows: 0, no staining; 1 , weak staining; 2 , moderate to strong staining. The percentage of positively stained cells was classified as follows: $0,<10 \%$; $1,10-50 \% ; 2,>50 \%$. The final intensity score was calculated by multiplying the staining intensity score by the staining percentage score. All cases were subsequently classified into the four expression groups according to the following final scores: 0 , negative $(-)$; 1 , weak $(+) ; 2$, moderate $(++) ; 3$, strong $(+++)$. Scores of ++ and +++ indicated positive GLUT-1 expression. To determine Ki-67 expression, positively stained cells were
Table I. Clinicopathological characteristics of pancreatic cancer patients $(\mathrm{n}=53)$.

\begin{tabular}{lc}
\hline Characteristics & Patients, n (\%) \\
\hline Age, years & 63 \\
Median & $39-72$ \\
Range & \\
Gender & $29(54.7)$ \\
Male & $24(45.3)$ \\
Female & \\
Tumor size, cm & 3.8 \\
Median & $1.1-7.4$ \\
Range & $18(34.0)$ \\
$\leq 2$ & $35(66.0)$ \\
$>2$ & \\
Differentiation & $13(24.5)$ \\
Well & $18(34.0)$ \\
Moderate & $22(41.5)$ \\
Poor & \\
Lymph node metastasis & $21(39.6)$ \\
Yes & $32(60.4)$ \\
No & \\
Clinical stage & $22(41.5)$ \\
II & $31(58.5)$ \\
\hline
\end{tabular}

defined as those exhibiting clear nuclear staining. Tissues were considered to exhibit positive Ki-67 expression when $>15 \%$ of the tumor cells were stained among $\geq 1,000$ tumor cells.

${ }^{18}$ F-FDG PET/computed tomography (CT). FDG-PET scans were performed on the 53 patients from mid-thigh to the head using a GE Discovery STE 16 PET/CT scanner (GE Healthcare, Piscataway, NJ, USA). Blood glucose levels were measured prior to ${ }^{18} \mathrm{~F}$-FDG injection, and patients with a blood glucose level of $>11.2 \mathrm{mmol} / 1$ were excluded from the study. Patients underwent FDG PET scans after $\geq 6 \mathrm{~h}$ fasting and an uptake time of 45-60 min following intravenous ${ }^{18} \mathrm{~F}$-FDG administration (3.70-4.44 MBq/kg). An emission scan was acquired for $3 \mathrm{~min}$ per bed position and a whole-body scan was performed for each patient using several bed positions, which were conducted based on the height of each patient.

The whole-body PET images were independently evaluated by two nuclear medicine physicians for the presence of abnormally increased uptake in the pancreas. PET, CT and fused PET/CT images were presented on a workstation to diagnose ${ }^{18} \mathrm{~F}$-FDG uptake in the pancreas. On the basis of regions of interest (ROIs), ${ }^{18}$ F-FDG uptake was analyzed semi-quantitatively by calculating the maximum standardized uptake value $\left(\mathrm{SUV}_{\max }\right)$ according to the following equation: $\mathrm{SUV}_{\max }=$ maximum pixel value within the ROI activity $(\mathrm{MBq} / \mathrm{kg}) /($ injected dose $[\mathrm{MBq}] /$ body weight $[\mathrm{kg}])$.

Statistical analysis. All statistical analyses were performed using SPSS 17.0 (SPSS, Inc., Chicago, IL, USA). Continuous 
Table II. Association between GLUT-1 expression and clinicopathological features of pancreatic cancer patients.

\begin{tabular}{|c|c|c|c|c|c|}
\hline \multirow[b]{2}{*}{ Parameter } & \multirow[b]{2}{*}{$\mathrm{n}$} & \multicolumn{2}{|c|}{ GLUT-1 expression } & \multirow[b]{2}{*}{$\chi^{2}$} & \multirow[b]{2}{*}{ P-value } \\
\hline & & Positive & Negative & & \\
\hline \multicolumn{6}{|l|}{ Gender } \\
\hline Male & 29 & 21 & 8 & 0.045 & 1.000 \\
\hline Female & 24 & 18 & 6 & & \\
\hline \multicolumn{6}{|l|}{ Age, years } \\
\hline$\leq 65$ & 28 & 19 & 9 & 1.002 & 0.365 \\
\hline$>65$ & 25 & 20 & 5 & & \\
\hline \multicolumn{6}{|l|}{ Tumor location ${ }^{\mathrm{b}}$} \\
\hline Head & 37 & 29 & 8 & 1.449 & 0.311 \\
\hline Body and tail & 16 & 10 & 6 & & \\
\hline \multicolumn{6}{|l|}{ Tumor size, cm } \\
\hline$\leq 2$ & 18 & 8 & 10 & 11.908 & 0.001 \\
\hline$>2$ & 35 & 31 & 4 & & \\
\hline \multicolumn{6}{|l|}{ Differentiation } \\
\hline Well & 13 & 8 & 5 & 1.287 & 0.525 \\
\hline Moderate & 18 & 14 & 4 & & \\
\hline Poor & 22 & 17 & 5 & & \\
\hline \multicolumn{6}{|l|}{ Clinical stage } \\
\hline I & 22 & 11 & 11 & 10.764 & $0.002^{\mathrm{a}}$ \\
\hline II & 31 & 28 & 3 & & \\
\hline \multicolumn{6}{|c|}{ Lymph node metastasis } \\
\hline $\mathrm{Y}$ & 21 & 19 & 2 & 5.105 & $0.029^{\mathrm{a}}$ \\
\hline $\mathrm{N}$ & 32 & 20 & 12 & & \\
\hline \multicolumn{6}{|c|}{ Vascular invasion } \\
\hline $\mathrm{Y}$ & 10 & 5 & 5 & 3.527 & 0.106 \\
\hline $\mathrm{N}$ & 43 & 34 & 9 & & \\
\hline
\end{tabular}

${ }^{\mathrm{a}} \mathrm{P}<0.05$. ${ }^{\mathrm{b}} \mathrm{Head}$, body and tail refer to the location of the tumor in the pancreas. GLUT-1, glucose transporter-1; Y, yes; N, no.

variables were compared using the Mann-Whitney U test and categorical variables were compared using the $\chi^{2}$ test or Fisher's exact test. The overall survival time was defined as the interval between the date of tumor resection and the date of mortality or last follow-up. Overall survival was calculated using the Kaplan-Meier method and compared by the log-rank test. Multivariate analysis was performed using the Cox proportional hazards regression model. Correlation analysis was performed using Spearman's rank analysis. Receiver operating characteristic (ROC) curve analysis was used to define a cut-off $\mathrm{SUV}_{\max }$ value for the optimal sensitivity and specificity in the prediction of GLUT-1 strong positive expression. $\mathrm{P}<0.05$ was considered to indicate a statistically significant difference.

\section{Results}

Overexpression of GLUT-1 protein in pancreatic cancer. To elucidate the function of GLUT-1 in the progression of pancreatic cancer, the expression of GLUT-1 protein in clinical pancreatic cancer tissues was analyzed using IHC staining. The GLUT-1 protein was predominantly localized to the cytomembrane of cancer cells in pancreatic cancer tissues (Fig. 1). Among the 53 pancreatic cancer tissues, 39 cases (73.6\%) exhibited positive GLUT-1 expression, including 25 strongly positive cases (47.2\%) in tumor tissues. Among the non-tumorous tissues, 42 cases (79.2\%) exhibited negative GLUT-1 expression and 11 cases exhibited positive expression (20.8\%). Thus, GLUT-1 expression was significantly higher in pancreatic cancer tissues when compared with non-tumor tissues $\left(\chi^{2}=29.681 ; \mathrm{P}<0.001\right)$.

Correlation between GLUT-1 protein expression and clinicopathological parameters. The associations between GLUT-1 expression and clinicopathological parameters of pancreatic cancer patients are shown in Table II. GLUT-1 expression significantly correlated with tumor size $\left(\chi^{2}=11.908 ; \mathrm{P}=0.001\right)$, clinical stage $\left(\chi^{2}=10.764 ; \mathrm{P}=0.002\right)$ and lymph node metastasis $\left(\chi^{2}=5.105 ; \mathrm{P}=0.029\right)$, however, no significant associations were identified between GLUT-1 expression and gender $\left(\chi^{2}=0.045\right.$; $\mathrm{P}=1.000)$, age $\left(\chi^{2}=1.002 ; \mathrm{P}=0.365\right)$, tumor location $\left(\chi^{2}=1.449\right.$; $\mathrm{P}=0.311)$, tumor differentiation $\left(\chi^{2}=1.287, \mathrm{P}=0.525\right)$ or vascular invasion $\left(\chi^{2}=3.527 ; \mathrm{P}=0.106\right)$. These results indicated that the 

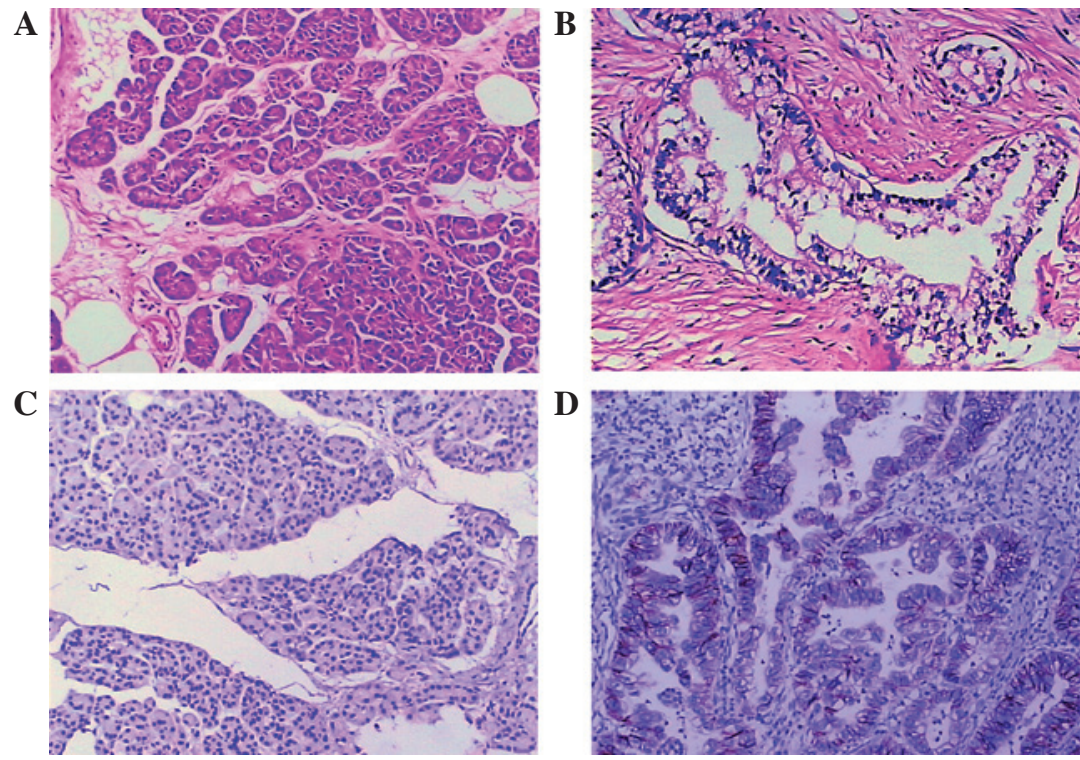

Figure 1. Representative immunohistochemical staining of GLUT-1. Hematoxylin and eosin staining of (A) pancreatic cancer tissues and (B) paired non-tumorous tissues, showing (C) negative GLUT-1 expression in non-tumorous tissues and (D) positive GLUT-1 expression in pancreatic cancer tissues. Magnification, x200. GLUT-1, glucose transporter-1.

overexpression of GLUT-1 may correlate with the progression of pancreatic cancer.

Prognostic significance of GLUT-1 overexpression. Of the 53 pancreatic cancer patients, 3 patients were lost to follow-up. As shown in Fig. 2, the median overall survival time for the GLUT-1 positive group was 12.3 months compared with 22.2 months for the GLUT-1 negative group. Kaplan-Meier curve analysis revealed that patients with positive GLUT-1 expression exhibited a significantly shorter overall survival time than those with GLUT-1 negative expression (log-rank test, $\mathrm{P}=0.001)$. Multivariate analysis revealed that GLUT-1 expression is an independent prognostic factor $(\mathrm{P}=0.001$; Table III). These results indicated that GLUT-1 overexpression is correlated with poor prognosis of pancreatic cancer.

Association between GLUT-1 expression and $S U V_{\max }$. All patients were examined by ${ }^{18} \mathrm{~F}-\mathrm{FDG}$ PET/CT. The median $\mathrm{SUV}_{\max }$ was 4.90 (range, 1.93-13.22; 25-75\% percentile, 2.96-7.04). As shown in Fig. 3A, the patients with positive GLUT-1 expression exhibited a significantly higher SUV $_{\max }$ than those exhibiting negative GLUT-1 expression (median $\mathrm{SUV}_{\max }, 6.07$ vs. 2.84; $\mathrm{P}<0.001$ ). In addition, Spearman's rank analysis indicated that $\mathrm{SUV}_{\max }$ is positively correlated with GLUT-1 expression in pancreatic cancer tissues $(\mathrm{r}=0.6885$; $\mathrm{P}<0.001$; Fig. 3B).

The sensitivity and specificity for the detection of GLUT-1 strong positive expression at different cutoff values of $S_{U} V_{\max }$ in pancreatic cancer patients were determined according to the ROC curve (Fig. 3C). A cutoff $S \mathrm{SV}_{\max }$ value of 4.830 exhibited the highest Youden's index (20) of 0.594, which was associated with optimal sensitivity (88\%) and specificity (71.4\%). The area under the ROC curve was 0.844 (95\% confidence interval, 0.7405-0.9480; $\mathrm{P}<0.001)$. According to the cutoff value, the 53 pancreatic cancer patients were divided into two groups: high and low $\mathrm{SUV}_{\max }$ groups. Among the 33 patients of the high $\mathrm{SUV}_{\max }$ group, 69.7\% (23/33) exhibited strong positive
GLUT-1 expression, while the remaining 30.3\% (10/33) of patients exhibited weak or moderate GLUT-1 expression. Of the 20 patients in the low $\mathrm{SUV}_{\max }$ group, 10\% (2/20) exhibited strong positive GLUT-1 expression, while 90\% (18/20) exhibited weak or moderate GLUT-1 expression. (Fig. 3D).

Association between GLUT-1 expression and Ki-67. To clarify the association between GLUT-1 and cell proliferation, the correlation between GLUT-1 and Ki-67 expression was examined in pancreatic cancer tissues (Fig. 4). Positive $\mathrm{Ki}-67$ expression was observed in $79.2 \%$ (42/53) of pancreatic cancer tissues and $22.7 \%(12 / 53)$ of adjacent non-tumorous tissues. Among the 53 tumor specimens, GLUT-1 expression was positively correlated with the Ki-67 expression ( $\mathrm{r}=0.327$; $\mathrm{P}=0.017$; Table IV).

\section{Discussion}

In the present study, the expression of GLUT-1 was examined in 53 pairs of paraffin-embedded pancreatic cancer tissues. The results revealed that GLUT-1 was overexpressed in pancreatic cancer tissues and its expression positively correlated with increased tumor size, higher clinical stage and lymph node metastasis. Additionally, GLUT-1 was identified as an independent prognostic factor for pancreatic cancer.

GLUT-1, a member of GLUT family, facilitates the entry of glucose across the plasma membrane. A number of studies have demonstrated a close association between GLUT-1 expression and malignant mesothelium, which is relevant for the clinical behavior of the tumor $(14,21,22)$. The results of the present study indicated that GLUT-1 was overexpressed in pancreatic cancer and was associated with clinicopathological characteristics, including tumor size, clinical stage and lymph node metastasis. In particular, the expression of GLUT-1 exhibited a significant effect on patient survival. Elevated GLUT-1 expression in tumor tissues reflects the requirement for a corresponding increase in glucose. Two possible 


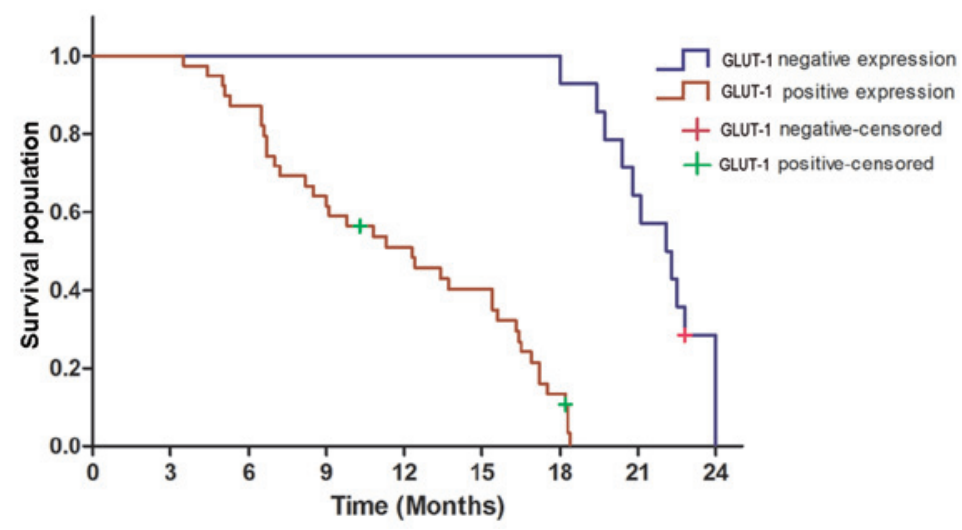

Figure 2. Kaplan-Meier survival curves comparing GLUT-1 expression in pancreatic cancer patients. The survival time in the GLUT-1 positive expression group was significantly shorter than that for patients in the GLUT-1 negative expression group ( $\mathrm{P}=0.001)$. GLUT-1, glucose transporter-1.
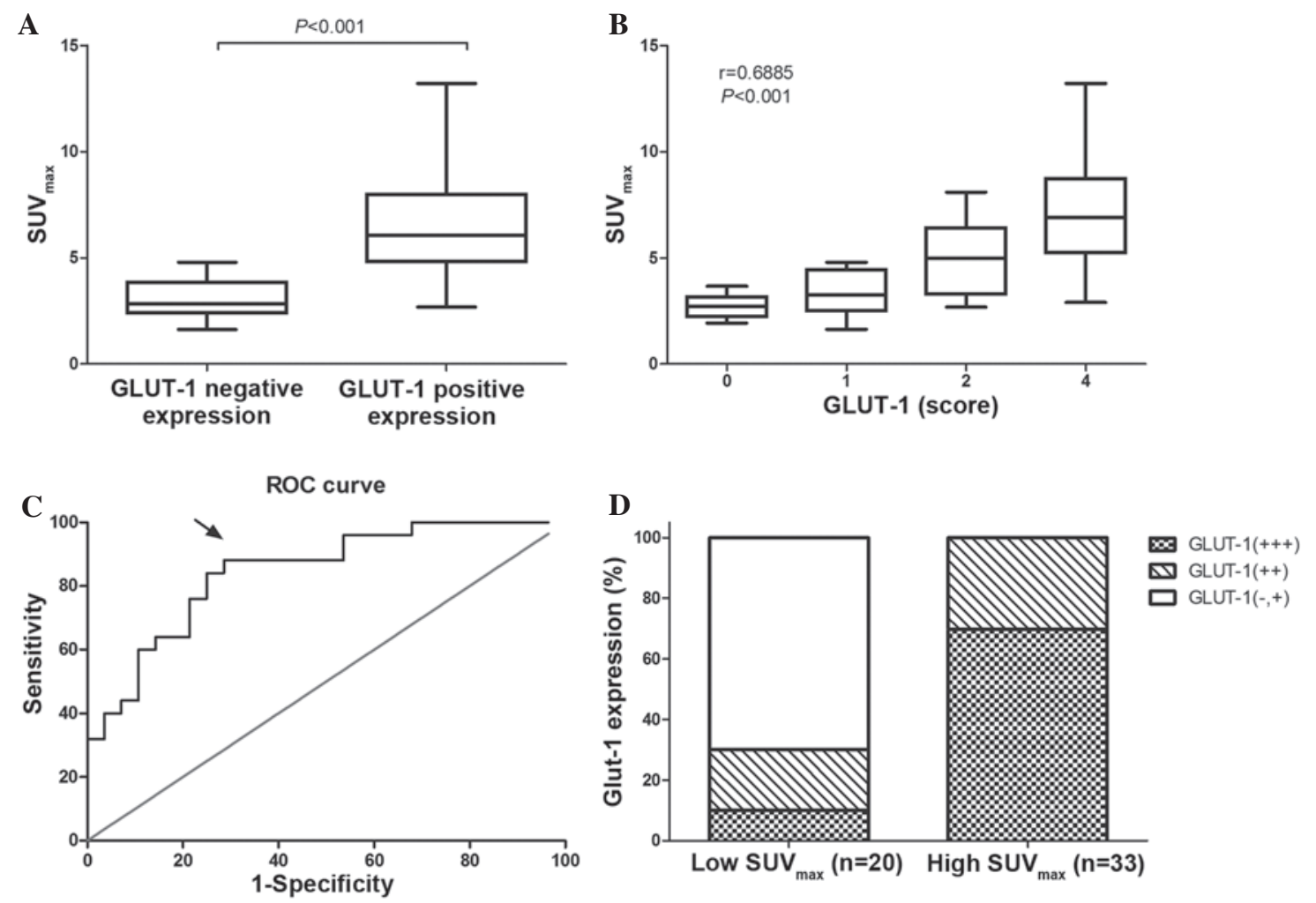

Figure 3. The association between GLUT-1 expression and $\mathrm{SUV}_{\max }$. (A) $\mathrm{SUV}_{\max }$ was measured by ${ }^{18} \mathrm{~F}$-fluorodeoxyglucose positron emission tomography/computed tomography. (B) The correlation between SUV max $_{\text {ax }}$ and GLUT-1 expression. (C) The sensitivity and specificity in the detection of GLUT-1 strong expression according to the ROC curve. The arrow indicates the optimal sensitivity (88\%) and specificity (71.4\%) at a cut-off SUV $\mathrm{V}_{\max }$ value of 4.830 . (D) Distribution of GLUT-1 expression according to the value of $\mathrm{SUV}_{\max }$. GLUT-1, glucose transporter-1; $\mathrm{SUV}_{\max }$, maximum standardized uptake value; ROC, receiver operating characteristic.
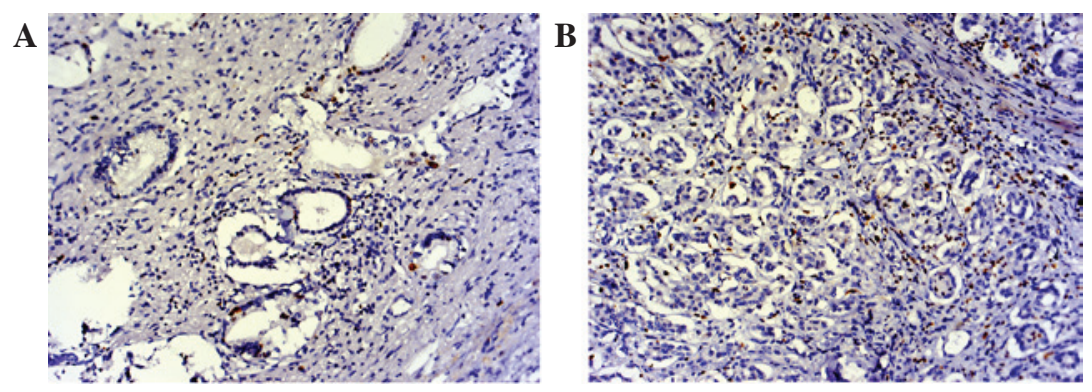

Figure 4. Expression of Ki-67 in two groups of pancreatic cancer tissues with (A) GLUT-1 negative expression and (B) GLUT-1 positive expression (magnification, x200). GLUT-1, glucose transporter-1. 
Table III. Multivariate analysis of prognostic markers in pancreatic cancer patients.

\begin{tabular}{lccr}
\hline Factors & HR & $95 \%$ CI & P-value \\
\hline Gender & 1.251 & $0.686-2.280$ & 0.466 \\
Age & 0.638 & $0.360-1.128$ & 0.122 \\
Tumor location & 1.385 & $0.690-2.778$ & 0.359 \\
Tumor size & 0.425 & $0.211-0.856$ & 0.017 \\
Differentiation & 1.426 & $0.697-2.915$ & 0.331 \\
Clinical stage & 0.537 & $0.306-0.943$ & 0.030 \\
Lymph node metastasis & 4.210 & $2.295-7.720$ & $<0.001$ \\
Vascular invasion & 0.583 & $0.302-1.125$ & 0.108 \\
GLUT-1 expression & 0.294 & $0.153-0.568$ & $<0.001$ \\
\hline
\end{tabular}

GLUT-1, glucose transporter-1; HR, hazard ratio; CI, confidence interval.

Table IV. Correlation between GLUT-1 and Ki-67 expression in pancreatic cancer patients.

\begin{tabular}{lcccrr}
\hline & \multicolumn{2}{c}{ GLUT-1 expression } & & \\
\cline { 2 - 3 } Ki-67 expression & Positive, $\mathrm{n}$ & Negative, $n$ & & $\mathrm{r}$ & P-value \\
\hline Positive, $\mathrm{n}$ & 34 & 8 & & \\
Negative, $\mathrm{n}$ & 5 & 6 & 0.327 & 0.017 \\
\hline
\end{tabular}

GLUT-1, glucose transporter-1.

mechanisms have been postulated to explain the overexpression of GLUT-1 in tumors. Firstly, local ischemia and hypoxia in the tumor may result in adaptive glycolytic metabolism and GLUT-1 expression (23). Secondly, GLUT-1 activity is widely upregulated via hypoxia-inducible factor-1 in hypoxic conditions $(24,25)$.

Certain factors affect FDG uptake, including hypoxia, cell density and expression of glycolysis-associated proteins $(26,27)$. In the present study, $\mathrm{SUV}_{\max }$ was significantly associated with the intensity of GLUT-1 expression and low GLUT-1 expression also corresponded to a low $\mathrm{SUV}_{\max }$. This may indicate that the sensitivity of $\mathrm{SUV}_{\max }$ for the pancreatic cancer patients with positive GLUT-1 expression is higher than those with negative expression. In ROC analysis, the positive and negative predictive values of $\mathrm{SUV}_{\max }$ for identifying GLUT-1 strong expression were 69.7\% (22/33) and 90\% $(18 / 20)$, respectively. We hypothesize that glucose consumption, as calculated by $\mathrm{SUV}_{\max }$ using ${ }^{18} \mathrm{~F}$-FDG/PET, predicted the level of GLUT-1 expression in pancreatic cancer patients. In addition, the cutoff value of $\mathrm{SUV}_{\max }$ may aid in the selection of patients for more aggressive gene therapy, particularly for advanced pancreatic cancer that is not suitable for resection.

In general, hypoxia leads to reduced proliferation and increased apoptosis. However, certain cancer cells in the hypoxic environment undergo adaptive changes and produce energy via anaerobic glycolysis, enabling their survival and proliferation $(28,29)$. Ki-67, a proliferation-related nuclear protein, is expressed in proliferating cells during all active phase of the cell cycle $(30,31)$. The results of the present study revealed a positive correlation between GLUT-1 expression and $\mathrm{Ki}-67$ expression. This indicates that proliferation and hypoxia are not exclusive, and that GLUT-1 may present a potential therapeutic target to limit glucose uptake, thereby limiting the proliferation of pancreatic cancer cells.

In conclusion, the present study demonstrated that the overexpression of GLUT-1 in pancreatic cancer tissues is significantly associated with the clinicopathological characteristics and prognosis of pancreatic cancer patients. In addition, the expression of GLUT-1 was positively associated with ${ }^{18} \mathrm{~F}$-FDG uptake and cell proliferation in pancreatic cancer. These findings suggest that GLUT-1 may present an underlying prognostic indicator and a potential therapeutic target for pancreatic cancer.

\section{Acknowledgements}

The present study was supported by the Project of Nature Science Foundation of China (grant no. 81201905), the Nature Science Research Grants at the University of Jiangsu Province of P.R. China (grant no. 14KJB320019) and the Project of Medical Research of Jiangsu Province (grant no. Q201402).

\section{References}

1. Siegel R, Naishadham D and Jemal A: Cancer statistics, 2013. CA Cancer J Clin 63: 11-30, 2013.

2. Ryan DP, Hong TS and Bardeesy N: Pancreatic adenocarcinoma. N Engl J Med 371: 1039-1049, 2014.

3. Vincent A, Herman J, Schulick R, Hruban RH and Goggins M: Pancreatic cancer. Lancet 378: 607-620, 2011.

4. Diener MK, Combs SE and Buchler MW: Chemoradiotherapy for locally advanced pancreatic cancer. Lancet Oncol 14:269-270, 2013.

5. Finger EC and Giaccia AJ: Hypoxia, inflammation, and the tumor microenvironment in metastatic disease. Cancer Metastasis Rev 29: 285-293, 2010.

6. Matsumoto S, Yasui H, Mitchell JB and Krishna MC: Imaging cycling tumor hypoxia. Cancer Res 70: 10019-10023, 2010.

7. Milane L, Ganesh S, Shah S, Duan ZF and Amiji M: Multi-modal strategies for overcoming tumor drug resistance: Hypoxia, the Warburg effect, stem cells, and multifunctional nanotechnology. J Control Release 155: 237-247, 2011.

8. Thorens B and Mueckler M: Glucose transporters in the 21st Century. Am J Physiol Endocrinol Metab 298: E141-E145, 2010.

9. Adekola K, Rosen ST and Shanmugam M: Glucose transporters in cancer metabolism. Curr Opin Oncol 24: 650-654, 2012.

10. Szablewski L: Expression of glucose transporters in cancers. Biochim Biophys Acta 1835: 164-169, 2013.

11. Krzeslak A, Wojcik-Krowiranda K, Forma E, Jozwiak P, Romanowicz H, Bienkiewicz A and Brys M: Expression of GLUT1 and GLUT3 glucose transporters in endometrial and breast cancers. Pathol Oncol Res 18: 721-728, 2012.

12. Wincewicz A, Sulkowska M, Koda M, Kanczuga-Koda L, Witkowska E and Sulkowski S: Significant coexpression of GLUT-1, Bcl-xL and Bax in colorectal cancer. Ann N Y Acad Sci 1095: 53-61, 2007.

13. Chiba I, Ogawa K, Morioka T, Shimoji H, Sunagawa N, Iraha S, Nishimaki T, Yoshimi N and Murayama S: Clinical significance of GLUT-1 expression in patients with esophageal cancer treated with concurrent chemoradiotherapy. Oncol Lett 2: 21-28, 2011.

14. Ohba S, Fujii H, Ito S, Fujimaki M, Matsumoto F, Furukawa M, Yokoyama J, Kusunoki T, Ikeda K and Hino O: Overexpression of GLUT-1 in the invasion front is associated with depth of oral squamous cell carcinoma and prognosis. J Oral Pathol Med 39: 74-78, 2010. 
15. Brophy S, Sheehan KM,McNamara DA, Deasy J, Bouchier-Hayes DJ and Kay EW: GLUT-1 expression and response to chemoradiotherapy in rectal cancer. Int J Cancer 125: 2778-2782, 2009.

16. Rastogi S, Banerjee S, Chellappan S and Simon GR: Glut-1 antibodies induce growth arrest and apoptosis in human cancer cell lines. Cancer Lett 257: 244-251, 2007.

17. Alakus H, Batur M, Schmidt M, Drebber U, Baldus SE, Vallböhmer D, Prenzel KL, Metzger R, Bollschweiler E, Hölscher AH and Mönig SP: Variable 18F-fluorodeoxyglucose uptake in gastric cancer is associated with different levels of GLUT-1 expression. Nucl Med Commun 31: 532-538, 2010.

18. Edge SB and Compton CC: The American Joint Committee on Cancer: The 7th edition of the AJCC cancer staging manual and the future of TNM. Ann Surg Oncol 17:1471-1474, 2010

19. Reynolds T: Declaration of Helsinki revised. J Natl Cancer Inst 92: 1801-1803, 2000.

20. Böhning D, Böhning W and Holling H: Revisiting Youden's index as a useful measure of the misclassification error in meta-analysis of diagnostic studies. Stat Methods Med Res 17: 543-554, 2008

21. Cho H, Lee YS, Kim J, Chung JY and Kim JH: Overexpression of glucose transporter-1 (GLUT-1) predicts poor prognosis in epithelial ovarian cancer. Cancer Invest 31: 607-615, 2013.

22. Sulkowska M, Wincewicz A, Sulkowski S, Koda M and Kanczuga-Koda L: Relations of TGF-beta1 with HIF-1 alpha, GLUT-1 and longer survival of colorectal cancer patients. Pathology 41: 254-260, 2009

23. Mayer A, Schmidt M, Seeger A, Serras AF, Vaupel P and Schmidberger H: GLUT-1 expression is largely unrelated to both hypoxia and the Warburg phenotype in squamous cell carcinomas of the vulva. BMC Cancer 14: 760, 2014.
24. Melstrom LG, Salabat MR, Ding XZ, Strouch MJ, Grippo PJ, Mirzoeva S, Pelling JC and Bentrem DJ: Apigenin down-regulates the hypoxia response genes: HIF-1 $\alpha$, GLUT-1 and VEGF in human pancreatic cancer cells. J Surg Res 167: 173-181, 2011.

25. Fraga A, Ribeiro R and Medeiros R: Tumor hypoxia: The role of HIF. Actas Urol Esp 33: 941-951, 2009.

26. Pugachev A, Ruan S, Carlin S, Larson SM, Campa J, Ling CC and Humm JL: Dependence of FDG uptake on tumor microenvironment. Int J Radiat Oncol Biol Phys 62: 545-553, 2005.

27. Huang T, Civelek AC, Li J, Jiang H, Ng CK, Postel GC, Shen B and Li XF: Tumor microenvironment-dependent 18F-FDG, 18F-fluorothymidine, and 18F-misonidazole uptake: A pilot study in mouse models of human non-small cell lung cancer. J Nucl Med 53: 1262-1268, 2012.

28. Jiang J, Tang YL and Liang XH: EMT: A new vision of hypoxia promoting cancer progression. Cancer Biol Ther 11: 714-723, 2011.

29. Osinsky S, Zavelevich M and Vaupel P: Tumor hypoxia and malignant progression. Exp Oncol 31: 80-86, 2009.

30. Lee HE, Kim MA, Lee BL and Kim WH: Low Ki-67 proliferation index is an indicator of poor prognosis in gastric cancer. J Surg Oncol 102: 201-206, 2010.

31. Viale G, Giobbie-Hurder A, Regan MM, Coates AS, Mastropasqua MG, Dell'Orto P, Maiorano E, MacGrogan G, Braye SG, Ohlschlegel C, et al: Prognostic and predictive value of centrally reviewed $\mathrm{Ki}-67$ labeling index in postmenopausal women with endocrine-responsive breast cancer: Results from breast international group trial 1-98 comparing adjuvant tamoxifen with letrozole. J Clin Oncol 26: 5569-5575, 2008. 